\title{
Where else would I look for it? A five-country qualitative study on purposes, strategies, and consequences of online health information seeking
}

\author{
Nicola Diviani, ${ }^{1,2}$ Eva Haukeland Fredriksen, ${ }^{3}$ Corine S. Meppelink, ${ }^{4}$ Judy Mullan, ${ }^{5}$ \\ Warren Rich, ${ }^{5}$ Tobba Therkildsen Sudmann ${ }^{3}$ \\ ${ }^{1}$ Department of Health Sciences and Health Policy, University of Lucerne, Switzerland; ${ }^{2}$ Swiss Paraplegic \\ Research, Nottwil, Switzerland; ${ }^{3}$ Western Norway University of Applied Sciences, Bergen, Norway; \\ ${ }^{4}$ Amsterdam School of Communication Research, University of Amsterdam, The Netherlands; \\ ${ }^{5}$ University of Wollongong, Australia
}

Significance for public health

Health care professionals should acknowledge patient's online health information (OHI) seeking behaviors and discuss this in their consultations, as people are hesitant to bring it up themselves. By incorporating $\mathrm{OHI}$ seeking in the consultations, professionals can utilize patient's desire to learn as a resource for treatment and empowerment, fostering at the same timeshared decision-making in healthcare.

\section{Abstract}

Background. Online health information (OHI) is widely available and consulted by many people in Western countries to gain health advice. The main goal of the present study is to provide a detailed account of the experiences among people from various demographic backgrounds living in high-income countries, who have used OHI.

Design and methods. Thematic analysis of 165 qualitative semi-structured interviews conducted among OHI users residing in Australia, Israel, the Netherlands, Norway, and Switzerland was performed.

Results. The lived experience of people using OHI seem not to differ across countries. The interviews show that searches for $\mathrm{OHI}$ are motivated from curiosity, sharing of experiences, or affirmation for actions already taken. Most people find it difficult to appraise the information, leading them to cross-check sources or discuss $\mathrm{OHI}$ with others. OHI seems to impact mostly some specific types of health behaviors, such as changes in diet or physical activity, while it only plays a complementary role for more serious health concerns. Participants often check OHI before seeing their GP, but are reluctant to discuss online content with health care personnel due to expected negative reception.

Conclusions. This study adds to the body of knowledge on eHealth literacy by demonstrating how $\mathrm{OHI}$ affects overall health behavior, strengthens patients' ability to understand, live with, and prepare themselves for diverse health challenges. The increasing digitalization of health communication and health care calls for further research on digital divides and patient-professional relations. Health care professionals should acknowledge OHI seeking and engage in discussions with patients to enable them to appreciate $\mathrm{OHI}$, and to support shared decision making in health care. The professionals can utilize patient's desire to learn as a resource for health prevention, promotion or treatment, and empowerment.

\section{Introduction}

Over the last decades, the traditional paternalistic approach to the doctor-patient relationship has been shifting towards a more patient-centered one, with patients increasingly becoming more actively involved in making decisions about their health. ${ }^{1}$ This has resulted in more patients seeking health information on the Internet to assist them in making informed decisions. ${ }^{2}$ Estimates suggest that three out of four Internet users worldwide have used it for health-related information. ${ }^{3}$ In this context, it is crucial that professionals in healthcare and public health are aware of the many challenges related to online health information (OHI) seeking. Ready access to OHI has proven to be beneficial in terms of improving patients' knowledge about different health issues. ${ }^{4}$ However, effective OHI seeking also requires a set of complex skills that not every individual will possess. OHI seeking is not only about finding information but also requires people to select it, understand it, evaluate it and decide whether and how to apply it. Some of these tasks are especially demanding. For instance, considering the often questionable quality of $\mathrm{OHI},{ }^{5-9}$ selecting the most appropriate website for information regarding a specific health issue can be particularly challenging. In such a context, people need to be able to evaluate the reliability and credibility of the websites they encounter.

One of the main risks associated with a lack of these essential skills is that people could be acting on wrong, irrelevant or incomplete information. This in turn could lead to negative consequences, such as poor health outcomes, as well as a deterioration in the doctor-patient relationship. ${ }^{10}$ Therefore, it is important that individuals possess the crucial skills that play a role in OHI seeking or, ${ }^{11,12}$ in other words, that they become more eHealth literate. ${ }^{13}$

Individual's OHI seeking has been studied in the past. Early studies applied a qualitative approach to explore the phenomenon and were mostly conducted among young and highly educated individuals in the United States or Europe because these were the population groups who mostly engaged in $\mathrm{OHI}$ seeking at that time. ${ }^{14-18}$ More recent studies investigating OHI seeking have used quantitative research designs, broadening the focus to a wider range of age groups and socio-economic backgrounds. These more recent studies have shown that OHI seeking has become a common practice among people of different ages, educational levels and socio-economic backgrounds across the world (e.g. Fox). ${ }^{3} \mathrm{OHI}$ seeking has changed over time and, therefore, 
cannot be considered a static phenomenon, thus deserving continuous in-depth investigations. ${ }^{19}$

Research on OHI seeking in general, and on eHealth literacy in particular, has mostly focused on its determinants, its prevalence, and its impact on health outcomes. For instance, we now know that younger people and people with a high educational level are more likely to possess higher eHealth literacy skills and to search for health information online (e.g. Tennant, Niederdeppe) ${ }^{20,21}$ Furthermore, possessing higher eHealth literacy skills is related to overall better experiences in OHI seeking and with increased knowledge. ${ }^{22}$ People's lived experience with $\mathrm{OHI}$ and how eHealth literacy skills are put into practice, on the other hand, has often been neglected.

This study aims to address this gap in the literature and to explore and describe the experiences with OHI seeking among people from different socio-economic backgrounds and living in various high-income countries across the globe. More specifically, this study aims to explore in-depth qualitative insights into the lived experience of applying eHealth literacy skills in different national contexts. This requires the provision of a detailed account of purposes, strategies and consequences of OHI seeking. This means including the whole process, from the detection of an itch or a worry or a curiosity which brings people to the keyboard, to the process or searching, to the evaluating of the information, and finally to the decision on what do next or, in other words, to the action part based on the OHI search. This new qualitative research would contribute to the existing body of knowledge on eHealth literacy and $\mathrm{OHI}$ seeking, particularly with the widespread use of digital technologies over the last decade. Countries included in this study differ in terms of languages spoken, availability of nationally quality assured OHI, the organization and funding of health care, and the development and implementation of eHealth.

\section{Design and Methods}

The present paper draws on qualitative data from a mixedmethod design study involving participants from five countries: Australia (AUS), Israel (IL), the Netherlands (NL), Norway (NO), and Switzerland $(\mathrm{CH})$. Using semi-structured in-depth interviews, this study explored the different processes involved in seeking or using OHI. By collecting detailed accounts of the activities performed online, we investigated whether and why people use OHI, their search strategies, how they evaluate the information found, and whether it affects health behaviors.

\section{Study design}

The study was conducted between 2014 and 2015 in Australia, Israel, the Netherlands, Norway, and Switzerland. This paper presents the results of 165 qualitative semi-structured interviews conducted among participants residing in these countries. Data from Australia, Israel, and the Netherlands were collected specifically for the present study. Data from Norway and Switzerland were collected in the context of larger ehealth research projects and results presented here are the results of a secondary comparative analysis of the original interviews. ${ }^{23,24}$ These original Swiss and Norwegian interviews have been_reanalyzed following the emergence of new themes across the different countries included in this study. A qualitative methodology was chosen because of the well-known value of qualitative methods in providing detailed accounts of people's experiences.

Data collection was approved by the Ticino Ethical Committee (ref. CE2773: Swiss data); the Amsterdam School of
Communication Research Ethics committee (ref. 2014-CW-126: Dutch and Israeli data); the Norwegian Social Science Data Services (ref. 40938: Norwegian data), and the University of Wollongong (ref. HE14/454: Australian data). All participants received a participant information sheet informing them about the study and were required to sign a consent form prior to their enrollment in the study. Participant information sheets and consent forms were translated by the researchers into the languages appropriate for each of the different countries included in the study.

\section{Study participants and recruitment}

Interviewers, in each country, used different techniques to recruit a heterogeneous convenience sample of participants. Interviewers in the different countries were instructed to fulfill quotas regarding gender, age and educational level. A snowball sampling technique was used to identify suitable interview participants who fulfilled the study criteria regarding specific sociodemographic profiles and who were willing to take part to the interview, except in the Netherlands, where potential participants were approached in the waiting area of a major international airport.

To be eligible for participation, participants were required to be over 18 years of age, to be able to speak and understand the respective national language, and to have previous experience with $\mathrm{OHI}$ seeking.

\section{Data collection}

A semi-structured interview guide was developed consisting of a series of open-ended questions. In order to mitigate recall bias, at the beginning of the interview, participants were asked to think about their most recent $\mathrm{OHI}$ search experience and to refer to that specific experience throughout the interview. The interview guide was developed using the different dimensions of the concept of eHealth literacy as a guide. ${ }^{13}$ Topics covered during the interview were motivations and purposes of the search; search strategies applied; problems encountered and related solutions; outcomes of the search; their perceptions about the quality of the OHI search and how they themselves assessed the quality of the OHI search; whether they discussed the OHI search with a healthcare provider or others (e.g., family, friends, or colleagues; and lastly how they decided to follow or ignore the advice found online.

The interview guide, originally developed in Italian to collect data from an Italian-speaking Swiss sample was first translated into English. ${ }^{24}$ The English and/or Italian version of the interview guide was subsequently translated into Dutch, Hebrew, and Norwegian by native speaking researchers of the respective countries, who also had a good command of English and/or Italian.

All interviews were conducted face-to-face except from the Israeli ones which were conducted via Skype. In Norway, the interviews were conducted by senior researchers, while in the other countries' interviews were conducted by research assistants and/or Master's students. The interviews lasted between 20 and $60 \mathrm{~min}-$ utes. Face-to-face interviews were longer than Skype, and senior researchers conducted longer interviews. In line with common practice in qualitative research, data collection in each country was ceased when additional interviews did not add new information. ${ }^{25}$

After the interviews, participants were asked to complete a short questionnaire including socio-demographic questions and the eHealth literacy scale. ${ }^{26}$

\section{Analyses}

Interviews were transcribed verbatim in the language in which they were conducted and analyzed using thematic analysis. The coding scheme developed for the Swiss data was used as a starting 
point to identify recurring themes and to ensure some level of consistency across analyses. ${ }^{24}$ When new themes or sub-themes emerged in one country they were promptly shared during regular Skype meetings among the research team so that everyone could include them in their analysis. Due to this iterative process, each interview was read several times. Each country produced a detailed summary of the main results including illustrative quotes translated into English for each of the identified themes and sub-themes. A comparison of the results from the different countries was conducted first by the national researchers and, subsequently, by the whole research team. The main differences and similarities across the countries were then summarized.

\section{Results}

Overall, slightly more than half of the 165 participants were women $(n=90,54.5 \%)$ and $54.5 \%(n=90)$ of the sample did not have a college education. The mean age of the participants was 42 years $(\mathrm{SD}=19.7)$. Average eHealth literacy scores within the country samples were similar, indicating an overall similar degree of confidence in the ability to interact with OHI. Detailed information about the socio-demographic characteristics of the participants are presented in Table 1.

Several common themes were identified across the different countries. In the following paragraphs, we will present a detailed account on the different aspects of purposes, strategies and consequences of OHI seeking. Overall, participants' accounts were highly homogeneous across the different countries, with only a few differences. Important differences between countries are highlighted throughout the analysis.

\section{Seeking and finding $\mathrm{OHI}$}

People from all countries used the Internet for many different health purposes, such as self-diagnosis or to avoid a GP visit. A participant from the Netherlands for instance stated:

«I was in the UK and my eye lid was exploding. So, I wondered what was going on» (Male, 25, NL).

In several cases, the Internet search was performed in conjunction with a GP visit, to prepare for it, to complement it, or - very rarely - to challenge it:

«If I went in there [the GP's office] without doing the research it would have just gone over my head, but because I did the research before it definitely helped me understand what he was trying to explain to me» (Male, 25, AUS)

«About the blood test I did, I wanted to see what the values mean, what it's all about» (Male, 53, IL)

«I searched for asthma. That was because I had to take asthma medication and I thought: Why is that? It appeared to be incorrect» (Female, 39, NL)

A desire to help others and/or simple curiosity about a health problem/topic discussed among their social networks or in the media were also often mentioned, for instance by an Australian participant:

«I've searched for my husband because he's getting some checks at the hospital next week» (Female, 45, AUS)

and a Norwegian participant who stated:

«I can sit and look up what everybody else suffers from. I have a mother with fibromyalgia. I did not know what this was and I have a brother with a staphylococcus infection in a leg [...] I search for one issue and then I go on and find more interesting information» (Female, 20, NO)

Several distinctive features of the Internet were mentioned by the participants as reasons to search for OHI. The most commonly cited were the unrestricted availability and accessibility of $\mathrm{OHI}$ and its ease of use, for example:

«Searching online responds to our need for quick information. We are all insecure, as a society I mean, we can't stand having to wait for something» (Female, 24, $\mathrm{CH}$ ).

An older participant in the Netherlands shared her opinion: «It's the easiest thing to do. You have to go through so many books before you have found it» (Female, 71, NL)

Interestingly, several participants seemed to consider the Internet as the only place where health information could be found. For instance, a man from Israel stated:

«Where else would I look for it?» (Male, 54, IL)

Issues related to privacy and sensitivity of information were only mentioned in Norway:

«This is not a problem you want to see a doctor for at once (bleeding from anus). So, it was to make a judgement. [...] To look for alternatives - to find out myself, without having to see a doctor» (Female, 35, NO)

Virtually all searches for OHI started from a single search engine, which in most cases was Google. Participants in the different countries also mentioned specific national, e.g., thuisarts.nl in the Netherlands and helsenorge.no in Norway, or international, e.g., WebMD, medical websites. None of the participants men-

Table 1. Overview of participants' socio-demographic characteristics.

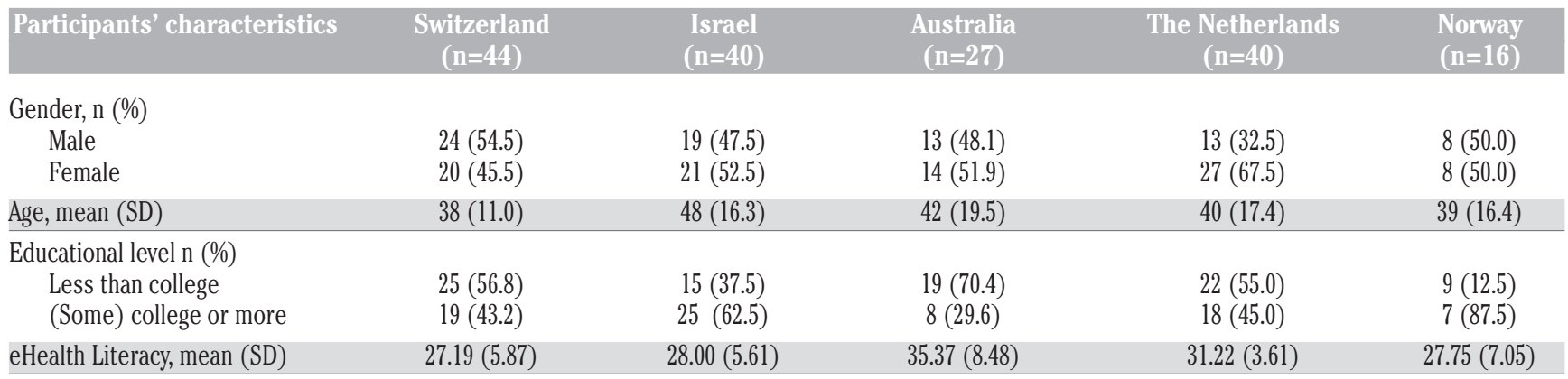


tioned having experienced technical problems, but some suggested that they did not know where to search for health information. Others mentioned having conducted the search to assist friends or family members who did not have the confidence and technical skills in undertaking the search themselves. A man from Switzerland, for example, reported:

«I did a search on prostate cancer for my uncle. I work in IT, so it is easier for me to search online and I know how to do it effectively» (Male, 23, $\mathrm{CH}$ )

Participants from non-English-speaking countries commented that since international search engines were better than their national ones, they used English terms, in addition to those in their native language to find more or better information. This contrasted with those with limited skills in English, who reported searching in English was a burden and preferred websites in their own language:

«Sometimes, there were entire websites in English that you had to read. My English is not so good which made me think: Oh, am I doing it correctly?» (Female, 61, NL)

Participants in all countries reported performing the online searches everywhere and at any time. Many of them used their readily available cell phones and often discussed their findings with family or friends. Some Norwegian participants reported that they performed searches together with other people and engaged in discussions during the search process:

«For example, when the low-carb diet was a big hype, we discussed much. Then we searched a lot together, and found articles of mutual interest: Look here...» (Female, 26, NO)

\section{Understanding and appraising $\mathrm{OHI}$}

Although many people seemed to search for health information on the Internet, understanding and appraising $\mathrm{OHI}$ appeared to be more difficult. Several participants, including highly educated ones, acknowledged that they sometimes had problems in understanding complex OHI, e.g., medical jargon, which resulted in terminating their search or not searching at all. As per the words of a participant from Switzerland:

"[I did a search] on tumors. But I did not go through with it. These are difficult things, you don't know what to search and it can get scary» (Male, $48, \mathrm{CH}$ )

Others disclosed that they did not know what health information to trust and that information overload scared them, which is why they preferred to receive health information directly from a physician:

«You know, in medicine 1 plus 1 could equal 11, so it is even more difficult to understand if the information is reliable or not» (Female, 24, $\mathrm{CH})$

A Norwegian participant undertook a kind of cost-benefit analysis regarding $\mathrm{OHI}$ :

"The uncertainty costs more than the symptoms themselves. I have learnt that if you go to the doctor first, you get rid of such worries quickly» (Male, 41, NO)

When participants were specifically asked about how they assess the quality of online information, several evaluation criteria were mentioned. There appeared to be consensus about the importance of the institution behind the website (information source) for the evaluation of the quality of a website. In three countries (the Netherlands, Israel and Norway) participants mentioned specific health websites that they trusted:
«Well, if you are looking at renowned websites, this is something different than a forum of blog written by some individual. This is less reliable than the website of the GGD [Dutch health authority] for example, which is quite reliable in my view» (Male, 28, NL)

In Israel, a woman told us about her preferred websites: "There are the sites of 'Maccabi' Health services, 'Clalit' Health services, those are obviously serious sites» (Female, 25, IL)

as did a Norwegian woman:

«There is not relevant information everywhere, but you can trust the official websites such as the Health Directorate and the Public Health Institute for example» (Female, 26, NO)

In the other countries, participants referred, more generally, to websites as "reliable sources" if they were from specific organisations, e.g., universities, governmental agencies, recognized associations:

«So I looked at things that were like university or government sites, like that, that I could perhaps be a little bit more sure that the information was legitimate» (Female, 60, AUS)

Some participants also mentioned searching for references to scientific publications when evaluating the quality of the website. For example, a participant from Australia mentioned:

«It always seems like if you have an article that's written by a doctor or if they have references and they can at least connect you with reviewed scientific literature then that's a good sign» (Male, 32, AUS)

Others suggested that they were less likely to trust commercial websites, especially when the authors were attempting to sell products:

«Some sites are obviously trying to promote a product, and the suspicion meter goes up» (Male, 55, IL)

The currency of the information, user friendliness, information complexity and clarity were other criteria used by the participants to assess the quality of OHI. For example, older websites and/or those not frequently updated were trusted less:

«The date, if it was dated I guess when you Google you can see the year it was published and if its dated information it will drive me away» (Male, 24, AUS)

Better quality was also attributed to websites that were easy to navigate and where information could be found quickly, as in the words of a woman in the Netherlands:

«It has to be readable on your phone, if not I go back directly, when you need to enlarge» (Female, 30, NL)

There was some disagreement among the study participants regarding their assessment of the quality of the website based on the complexity of the information. For some, complex information, e.g., medical concepts, was perceived to be more reliable:

«It's probably in terms of explanation quite good, it's probably

a little bit done in layman's referencing way and explains it quite simply» (Male, 70, AUS)

Others, however, held a different opinion:

«[I stop reading] When it consists of many difficult words, and I don't understand a thing of it» (Female, 56, NL) 
Many participants from the different countries mentioned using appearance, layout and writing style as indicators of the quality of a website. For example, a young Dutch male, when asked to indicate what makes him decide if a website is of good quality, simply stated:

«Whether it looks pretty» (Male, 24, NL)

Many also reported using the ranking of the search results, or its perceived popularity, i.e., the perceived number of users:

«Yes, the order that is presented by Google. This makes it more reliable to me. Maybe it's not, but it is viewed more often. Or they do a better job at their IT departments» (Female, 30, NL)

Some perceived the presence of chat room, forums, or comment sections on the website as quality indicators, while others perceived the presence of these interactive features as an indication of poor quality, as suggested by a participant from the Netherlands:

«Sometimes there are forums or blogs which make me think: well, this is quite one-sided information. Those are not the sources I use for information. But I use them sometimes to see how others deal with certain things» (Female, 28, NL)

Some started their search to look for other persons 'experiences, symptoms and concerns, to confirm their own queries and obtain ideas for relevant search concepts and strategies on trusted websites. The information found was compared within and between different websites and platforms until they reached a status of satisfaction about the website's reliability:

«I clicked into five, perhaps as much as ten websites, and checked what different sites wrote about the symptoms, to confirm my knowledge. Then I returned to the sites which looked most trustworthy, and read about treatment and what I could do myself» (Male, 26, NO)

\section{Applying the information obtained to address or solve a health problem}

The last question addressed in our study concerned the use of OHI. We found participants used different strategies when applying the obtained information, with and without involving a doctor. First, some participants reported not contacting a doctor or modifying their health behaviors after their OHI searches. We interpreted this as an active application of knowledge found on the different websites as support to actions already taken, or refraining from further actions. Participants who modified their health behaviors, based on the OHI, often changed their diet or workout-related habits. For example, a participant from Israel stated:

«I am a vegan now, I don't eat meat nor dairy products nor eggs and other things like that $[\ldots] \gg$ (Female, 56, IL)

None of the participants reported changing their medication taking or other health related behavior based solely on the $\mathrm{OHI}$ without consulting their doctor first. Among those who visited a doctor after an online search some did not mention their $\mathrm{OHI}$ searches, mostly because they were worried about a possible negative reaction from the doctor:

«When the doctor told me I had a specific disease, I went online to read about it. The next day when I came to talk to her and ask questions about it, I told her that I read on the Internet. Her response was: 'Never mind the Internet, listen to me'» (Male, 58, IL)

«[I don't discuss my online searches with my doctor] because he would throw me out the window» (Female, 70, $\mathrm{CH}$ )
Others prepared themselves through online searches and discussed their findings with their doctor. A few did not trust the doctor to know enough about their condition and even prepared themselves to teach them. Several of these strategies were combined with new searches after the medical encounter to confirm medical decisions and treatment plans before adhering to them.

\section{Discussion}

This study aimed to describe the lived experience of seeking, appraising and applying OHI among people from different sociodemographic backgrounds and living in five high-income countries across the globe. The large number and the diversity of the people who participated to the semi-structured interviews allowed us to draw a general picture on the phenomenon and, at the same time, to compare specific experiences and preferences across contexts. Generally, OHI affects overall health behavior, strengthens patients' ability to understand, live with, and prepare themselves for diverse health challenges. It is also about agency, empowerment, and curiosity. Although our objective was not specifically to compare OHI seeking behaviors across different countries or socio-economic backgrounds, we were surprised to find that there were remarkable similarities in how people search for OHI across the different countries and across socio-economic groups. This is an important insight, as it provides evidence supporting the applicability of the results research on OHI seeking across contexts.

First, our study showed that in all the participating highincome countries, people frequently search for health information online because of its ease of use and availability. Physical accessibility of $\mathrm{OHI}$ does not seem to be a problem. Irrespective of the country people lived in, they mostly used Google as the starting point for their information search. This is supported by Fox and Duggan, ${ }^{3}$ who reported that $77 \%$ of American OHI searches commenced with a general search engine. The extensive use of a search engine, as a starting point, is not remarkable because many people reported searching for reasons of curiosity or in connection with visiting a GP with specific symptoms. It is reasonable to assume a previously healthy person would not have knowledge about specific disease-related websites. This finding emphasizes the importance of authoritative and quality-controlled websites to construct their pages in accordance with how Google or another search engines compute and create their page rank.

Second, although consulting the Internet for health information seems to be a common practice, people often find it difficult to understand the information they encounter and evaluate its reliability. This was mainly due to the frequent use of complex medical terminology or information in a language which may have differed from their mother tongue. This finding corresponds to the conclusion drawn by several studies stating that, generally, $\mathrm{OHI}$ is still at a level that is too difficult for many people to understand. ${ }^{23,28,29}$

Responses regarding the quality and reliability of $\mathrm{OHI}$ showed that it was mainly the institution behind the site that was trusted. However, while the source of the OHI is important to our participants, it may be difficult to judge the accuracy of the information. Furthermore, many also reported that they usually rely upon superficial aspects of the site, such as appearance, to judge credibility. The use of both official criteria, such as information source, and subjective ones, such a layout or Google ranking, are in line with a previous study. ${ }^{24}$ This finding emphasizes the need to improve consumers' ability to evaluate $\mathrm{OHI}$ by recommending the use of official quality criteria. ${ }^{30}$

The finding that most people are generally skeptical about the overall quality of $\mathrm{OHI}$ also relates to the third important finding of 
our study. Although many people performed OHI searches to find support for their decisions, they rarely modified their behavior and appeared to exercise a great deal of caution before acting upon OHI. While this could also mean that people do not trust the online information, this aspect was hardly mentioned by our participants. The lack of confidence in the quality and the relevance of $\mathrm{OHI}$ could also explain why many searched for $\mathrm{OHI}$ in conjunction with a visit to their GP, even though they sometimes chose not to discuss their searches during the consultation. They may have decided not to discuss their OHI searches with the GP because they were afraid that the GP would not take them seriously or because they believed that the information that they had obtained online would not add anything to the consultation. Healthcare professionals should therefore be more encouraging to discuss OHI seeking during their consultations. They should endeavor to be less judgmental regarding OHI use by their patients and further support their patients by suggesting reliable OHI sources and making OHI part of the consultation.

The latter result points to a trend that we observed, namely health information seeking as a collective, rather than individual, practice. This particularly refers to the Norwegian data. Future research is needed to investigate whether this collective OHI seeking also takes place in other countries and on what scale. If so, this could have important implications for both research and practice. From a research perspective, it would require shifting the attention from individual skills to the social context. From this social perspective, existing measures such as the eHealth Literacy Scale, ${ }^{26}$ would not be suitable to fully capture the phenomenon of $\mathrm{OHI}$ seeking. From a practical perspective, it would compel researchers and health care professionals to consider the patient's environment when assessing people's eHealth literacy skills.

We acknowledge that our study has some limitations. First, the composition of the research team and differences between the national researchers (male $v s$. female; senior $v s$. junior), as well as differences in type and location of data collection (face-to-face $v s$. Skype interviews; strangers approached at an airport vs. persons reached through snowballing), might have influenced our data collection and, ultimately, the research findings. All these factors are well-known to have a potential effect on the outcome of a study. ${ }^{31}$ The similarities in the results across countries, despite differences in data collection, can therefore be an indicator of the validity of our findings. A second limitation of our study is related to its retrospective nature and to the consequent reliance on participants' recall of past experiences (although in some cases they performed real time searches in front of the researchers to illustrate how they navigated the whole process). This might have resulted in incomplete or selective reporting. A further limitation resides in the choice of the countries to be included in the study. A more diverse selection of countries, for instance, including non-western and low-income countries, might have led to different results and likely to the identification of clear cross-country differences. Finally, our qualitative methodology does not allow us to generalize our findings beyond our study population. However, our aim was not to get a representative picture of the populations in the five countries but rather to get detailed accounts of their experiences. The findings from this study are relevant in terms of understanding OHI behavior in high-income countries, and the knowledge claims we make contribute to the existing body of knowledge. As for other qualitative studies, the application of our findings by the international scholar community or in e-health promotion campaigns are the real test of the study's knowledge claims.

\section{Conclusions}

This study adds to the body of knowledge on eHealth literacy by demonstrating that health professionals must be curious about the patients' online health behaviors and contribute to their empowerment, agency and eHealth literacy. Our study demonstrates that people frequently search for OHI, but find it difficult to understand and evaluate $\mathrm{OHI}$ 's reliability. $\mathrm{OHI}$ is rarely used as a basis to modify current health behaviors, but rather to find support for decisions already made. People seem to exercise a great deal of caution before acting upon OHI, but also before discussing their OHI seeking with a health professional. These two facts have important implications on how we understand OHI seeking, as they could indicate that its potential for harmful consequences is not as big as it has often been described. ${ }^{32}$ The potential health

Correspondence: Nicola Diviani,Department of Health Sciences and Health Policy, University of Lucerne Frohburgstrasse 3, 6002 Lucerne, Switzerland. Tel.: +41.41.2295634 - Fax: +41.41.2295001.

E-mail: nicola.diviani@unilu.ch

Key words: Health communication; Health information seeking; Online health information; eHealth literacy; Qualitative methods.

Contributions: All authors contributed to the conception of the work and to the acquisition, analysis, and interpretation of data. ND drafted the first version of the manuscript and all co-authors contributed to its critical revision. All authors approved the final version to be published. EHF, CSM, JM, WR, and TTS are listed in alphabetical order and contributed equally to the manuscript.

Conflict of interest: the authors declare no potential conflict of interest.

Acknowledgements: this paper is the result of an international collaboration started during the CPC/WUN Health Literacy Network Collaborative Workshop held in September 2014 in Amsterdam, The Netherlands. An earlier version of the paper was presented at the 14th International Conference on Communication in Healthcare - EACH held in September 2016 in Heidelberg, Germany. The authors wish to thank Remco Sanders and Keshet Katz for their valuable help in the recruitment of participants and in the data collection in The Netherlands and Israel.

Funding: this work was mainly supported by a grant by the Swiss National Science Foundation [study design and data collection in Switzerland: grant number P2TIP1_148345]. Data collection in the other countries was supported by small grants by the Amsterdam School of Communication Research, University of Amsterdam [data collection in the Netherlands and Israel]; University of Wollongong [data collection in Australia]; Western Norway University of Applied Sciences [data collection in Norway]. The funding sources had no role in study design, data collection, analysis, interpretation, or writing of the report.

Received for publication: 7 November 2018.

Revision received: 26 February 2019.

Accepted for publication: 1 March 2019.

(C) Copyright N. Diviani et al., 2019

Licensee PAGEPress, Italy

Journal of Public Health Research 2019;8:1518

doi:10.4081/jphr.2019.1518

This work is licensed under a Creative Commons Attribution NonCommercial 4.0 License (CC BY-NC 4.0).

benefits of $\mathrm{OHI}$ would similarly be lost, if health professionals do not take into consideration and/or appreciate that many people do engage with $\mathrm{OHI}$ seeking behaviours. 


\section{References}

1. Charles C, Gafni A, Whelan T. Shared decision-making in the medical encounter: what does it mean? (or it takes at least two to tango). Soc Sci Med 1997;44:681-92.

2. Dahl S, Dahl S, Eagle L, Eagle L. Empowering or misleading? Online health information provision challenges. Mark Intell Plan 2016;34:1000-20.

3. Fox S, Duggan M. Health online 2013. Health (NY) 2013;155.

4. Hardey M. "E-health": the internet and the transformation of patients into consumers and producers of health knowledge. Inf Commun Soc 2001;4:388-405.

5. Berland GK, Elliott MN, Morales LS, et al. Health information on the Internet: accessibility, quality, and readability in English and Spanish. JAMA 2001;285:2612-21.

6. Bernstam EV, Walji MF, Sagaram S, et al. Commonly cited website quality criteria are not effective at identifying inaccurate online information about breast cancer. Cancer 2008;112:1206-13.

7. Eysenbach G, Powell J, Kuss O, Sa ER. Empirical studies assessing the quality of health information for consumers on the world wide web: a systematic review. JAMA 2002;287:2691-700.

8. Sak G, Diviani N, Allam A, Schulz PJ. Comparing the quality of pro-and anti-vaccination online information: a content analysis of vaccination-related webpages. BMC Public Health 2016;16:1.

9. Stvilia B, Mon L, Yi YJ. A model for online consumer health information quality. J Am Soc Inf Sci Technol 2009;60:178191.

10. Cline RJ, Haynes KM. Consumer health information seeking on the Internet: the state of the art. Health Educ Res 2001;16:671-92.

11. Diviani N, van den Putte B, Giani S, van Weert JC. Low health literacy and evaluation of online health information: a systematic review of the literature. J Med Internet Res 2015;17.

12. Ye Y. Correlates of consumer trust in online health information: findings from the health information national trends survey. J Health Commun 2010;16:34-49.

13. Norman CD, Skinner HA. eHealth literacy: essential skills for consumer health in a networked world. J Med Internet Res 2006;8:e9.

14. Bernhardt JM, Felter EM. Online pediatric information seeking among mothers of young children: results from a qualitative study using focus groups. J Med Internet Res 2004;6.

15. Eysenbach G, Köhler C. How do consumers search for and appraise health information on the world wide web? Qualitative study using focus groups, usability tests, and indepth interviews. BMJ 2002;324:573-7.

16. Gray NJ, Klein JD, Noyce PR, et al. Health information-seeking behaviour in adolescence: the place of the internet. Soc Sci
Med 2005;60:1467-78

17. Hansen DL, Derry HA, Resnick PJ, Richardson CR. Adolescents searching for health information on the Internet: an observational study. J Med Internet Res 2003;5.

18. Skinner H, Biscope S, Poland B, Goldberg E. How adolescents use technology for health information: implications for health professionals from focus group studies. J Med Internet Res 2003;5.

19. Powell J, Inglis N, Ronnie J, Large S. The Characteristics and Motivations of Online Health Information Seekers: CrossSectional Survey and Qualitative Interview Study. J Med Internet Res 2011;13.

20. Tennant B, Stellefson M, Dodd V, et al. eHealth Literacy and Web 2.0 Health Information Seeking Behaviors Among Baby Boomers and Older Adults. J Med Internet Res 2015;17.

21. Niederdeppe J, Hornik RC, Kelly BJ, et al. Examining the dimensions of cancer-related information seeking and scanning behavior. Health Commun 2007;22:153-67.

22. Mitsutake S, Shibata A, Ishii K, Oka K. Association of eHealth Literacy With Colorectal Cancer Knowledge and Screening Practice Among Internet Users in Japan. J Med Internet Res 2012;14.

23. Fredriksen EH, Sudmann TT. Fra søk til bruk av helseinformasjon på Internett: e-Health literacy i en norsk kontekst [From seeking to using Internet-based health information: e-health literacy in a Norwegian context]. Fysioterapeuten 2016;83:1621.

24. Diviani N, van den Putte B, Meppelink CS, van Weert JC. Exploring the role of health literacy in the evaluation of online health information: Insights from a mixed-methods study. Patient Educ Couns 2016.

25. Guest G, Bunce A, Johnson L. How many interviews are enough? An experiment with data saturation and variability. Field Methods 2006;18:59-82.

26. Norman CD, Skinner H. eHEALS: The eHealth Literacy Scale. J Med Internet Res 2006;8.

27. Friemel TN. The digital divide has grown old: Determinants of a digital divide among seniors. New Media Soc 2016;18:313331.

28. Kim H, Xie B. Health literacy in the eHealth era: A systematic review of the literature. Patient Educ Couns 2017;100:107382.

29. Meppelink CS, van Weert JC, Brosius A, Smit EG. Dutch Health Websites and their Ability to Inform People with Low Health Literacy. Patient Educ Couns 2017;100:2012-9.

30. Diviani N, Meppelink CS. The impact of recommendations and warnings on the quality evaluation of health websites: An online experiment. Comput Hum Behav 2017;71:122-9.

31. Denscombe M. The good research guide: for small-scale social research projects. New York: McGraw-Hill Education; 2014.

32. Benigeri M, Pluye P. Shortcomings of health information on the Internet. Health Promot Int 2003;18:381-6. 